\title{
Childhood Ganglioneuroblastoma, Intermixed
}

National Cancer Institute

\section{Source}

National Cancer Institute. Childhood Ganglioneuroblastoma, Intermixed. NCI Thesaurus.

Code C124273.

An intermixed gang lioneuroblastoma that occurs during childhood. 